\title{
The Evolution of Payload Data Capabilities on the Commercial Visiting Vehicles that Service the International Space Station
}

\author{
Lindsay M. Wiggins ${ }^{1}$ and Ivica 'Vinny’ Ristovski \\ ${ }^{1}$ NASA Marshall Space Flight Center, Huntsville, AL., 35812, U.S., Lindsay.Wiggins@ nasa.gov \\ ${ }^{2}$ COLSA Corporation, Huntsville, AL., 35806, U.S., Ivica.Ristovski@nasa.gov
}

In 2008, NASA awarded the first contracts to U.S. commercial companies to deliver cargo and supplies to the International Space Station (ISS). These contracts, called the first phase of Commercial Resupply Services (CRS1), were awarded to Space Exploration Technologies (SpaceX) and Orbital Sciences. Under the CRS1 contracts, commercial visiting vehicles not only provide a couple tons of cargo to the ISS each mission, but they also provide the ability for payloads to be transferred to the ISS in an active, powered state. Prior to the start of the CRS1 program, most vehicles that serviced the ISS transported science experiments as passive cargo. Therefore, the CRS1 program through offering frequent flight opportunities with powered payload transport capability ushered in a new era in which NASA and payload developers could reimagine operational concepts for payloads during the transit phase to and from the ISS.

To take advantage of this powered payload transport capability, NASA first added requirements under the CRS1 program for the commercial vehicles to provide telemetry monitoring services for pressurized payloads to give payload developers situational awareness during the transport phase. Since then, payload developer use-cases for visiting vehicle data services during free-flight have evolved with each new commercial visiting vehicle contract to cover a variety of payload monitoring and control abilities. Additional commercial contracts include the Commercial Crew Transportation Capability (CCtCap) contracts that were awarded to SpaceX and Boeing, and the second phase of Commercial Resupply Services (CRS2) contracts that were awarded to SpaceX, Orbital ATK (formerly Orbital Sciences), and Sierra Nevada Corporation. Commercial crew flights will commence in 2018, and the first flight under the CRS2 program is currently planned for 2019.

As new requirements have been added on to commercial visiting vehicle contracts for payload data services over the years, the Payload Operations Integration Center (POIC) at the NASA Marshall Space Flight Center has made many payload ground system changes to enable payload developers to use these capabilities. To start, under CRS1, the POIC developed interfaces with commercial partners to enable payload developers to receive pressurized payload health and status telemetry as well as some real-time visiting vehicle telemetry including cabin $\mathrm{CO}_{2}$ and $\mathrm{O}_{2}$ content for insight into the environmental conditions within the vehicle.

The POIC also plans to make many more ground system changes to accommodate new services in the future. Under the CCtCap program, the POIC is establishing a new capability with the commercial visiting vehicle providers for payload developers to retrieve playback visiting vehicle environment data. For this capability, commercial providers will record visiting vehicle environment data on board during loss-of-signal (LOS) periods and provide the telemetry to payload developers through the POIC to support post flight data analysis. Lastly, under the new CRS2 program, the POIC is working with the commercial visiting vehicle providers to offer payload command services to pressurized payloads as well as state monitoring and control capabilities for unpressurized payloads during transit.

The goal for the new CRS2 payload commanding capability is to enable payload developers to use Internet Protocol (IP) services including command line protocols during the transit phase to make payload configuration changes and to turn payload equipment on and off. The intent is for this service to support payload developer use of Internet Control Message Protocol (ICMP), User Datagram Protocol (UDP), and Transmission Control Protocol (TCP) services. The greatest challenge in providing this capability to payloads is getting enough bandwidth allocated on the visiting vehicle during free-flight to enable successful use of bi-directional IP services. The goal for the new CRS2 unpressurized payload capabilities is to provide cyclic health and status data to payload developers and to establish analog and discrete line interfaces between unpressurized payloads and each visiting vehicle so payload developers can monitor temperatures at the payload site and do basic payload control. This paper will first detail the data services that will be available to payloads under each commercial 
visiting vehicle contract. Secondly, this paper will offer discussion of the most significant technical challenges faced to-date in offering these new data services to payloads.

As the POIC works through these challenges with each commercial visiting vehicle provider to offer new data services to the ISS payload developer community, there are three objectives that must be kept in balance. The first objective is to keep POIC ground system development costs low by standardizing implementation approaches for these data services across commercial vehicles. Standardization will reduce the amount of custom interface software that the POIC has to develop. At the same time, another goal is for these payload services to be implemented in a manner that is cost effective for each commercial visiting vehicle partner. Lastly, payloads are designed to operate on the ISS, so a third objective is for the interfaces that the commercial visiting vehicles provide on board for payloads to be consistent with the interfaces payloads have on the ISS to enable scientists to use these new data services without needing to make extensive payload hardware or software changes.

Therefore, as new capabilities are developed for Commercial Crew and CRS2 missions in the future, the POIC will strive to balance these competing objectives to deliver the capabilities that NASA payload developers need to ensure operational success once payloads are transferred to ISS in a manner that is both cost effective for all parties and meaningful in supporting the operational concepts payload developers have today. This paper will lastly provide discussion of the programmatic challenges related to balancing the impacts for new payload data services across the commercial visiting vehicle partners, the POIC, and the payload developer community. 